\title{
Cancer risk estimates from the combined Japanese A-bomb and Hodgkin cohorts for doses relevant to radiotherapy
}

\author{
Schneider, Uwe ; Walsh, Linda
}

\begin{abstract}
Most information on the dose-response of radiation-induced cancer is derived from data on the A-bomb survivors who were exposed to -rays and neutrons. Since, for radiation protection purposes, the dose span of main interest is between 0 and $1 \mathrm{~Gy}$, the analysis of the A-bomb survivors is usually focused on this range. However, estimates of cancer risk for doses above $1 \mathrm{~Gy}$ are becoming more important for radiotherapy patients and for long-term manned missions in space research. Therefore in this work, emphasis is placed on doses relevant for radiotherapy with respect to radiation-induced solid cancer. The analysis of the A-bomb survivor's data was extended by including two extra high-dose categories (4-6Sv and 6-13Sv) and by an attempted combination with cancer data on patients receiving radiotherapy for Hodgkin's disease. In addition, since there are some recent indications for a high neutron dose contribution, the data were fitted separately for three different values for the relative biological effectiveness (RBE) of the neutrons (10, 35 and 100) and a variable RBE as a function of dose. The data were fitted using a linear, a linear-exponential and a plateau-dose-response relationship. Best agreement was found for the plateau model with a dose-varying RBE. It can be concluded that for doses above 1Gy there is a tendency for a nonlinear dose-response curve. In addition, there is evidence of a neutron RBE greater than 10 for the A-bomb survivor data. Many problems and uncertainties are involved in combing these two datasets. However, since very little is currently known about the shape of dose-response relationships for radiation-induced cancer in the radiotherapy dose range, this approach could be regarded as a first attempt to acquire more information on this area. The work presented here also provides the first direct evidence that the bending over of the solid cancer excess risk dose response curve for the A-bomb survivors, generally observed above $2 \mathrm{~Gy}$, is due to cell killing effects
\end{abstract}

DOI: https://doi.org/10.1007/s00411-007-0151-y

Posted at the Zurich Open Repository and Archive, University of Zurich ZORA URL: https://doi.org/10.5167/uzh-156473

Journal Article

Published Version

Originally published at:

Schneider, Uwe; Walsh, Linda (2008). Cancer risk estimates from the combined Japanese A-bomb and Hodgkin cohorts for doses relevant to radiotherapy. Radiation and Environmental Biophysics, 47(2):253263.

DOI: https://doi.org/10.1007/s00411-007-0151-y 


\title{
Cancer risk estimates from the combined Japanese A-bomb and Hodgkin cohorts for doses relevant to radiotherapy
}

\author{
Uwe Schneider · Linda Walsh
}

Received: 1 November 2006/Accepted: 6 December 2007/Published online: 21 December 2007

(C) Springer-Verlag 2007

\begin{abstract}
Most information on the dose-response of radiation-induced cancer is derived from data on the A-bomb survivors who were exposed to $\gamma$-rays and neutrons. Since, for radiation protection purposes, the dose span of main interest is between 0 and $1 \mathrm{~Gy}$, the analysis of the A-bomb survivors is usually focused on this range. However, estimates of cancer risk for doses above $1 \mathrm{~Gy}$ are becoming more important for radiotherapy patients and for long-term manned missions in space research. Therefore in this work, emphasis is placed on doses relevant for radiotherapy with respect to radiation-induced solid cancer. The analysis of the A-bomb survivor's data was extended by including two extra high-dose categories (4-6 Sv and 6-13 Sv) and by an attempted combination with cancer data on patients receiving radiotherapy for Hodgkin's disease. In addition, since there are some recent indications for a high neutron dose contribution, the data were fitted separately for three different values for the relative biological effectiveness (RBE) of the neutrons (10, 35 and 100 ) and a variable RBE as a function of dose. The data were fitted using a linear, a linear-exponential and a plateau-dose-response relationship. Best agreement was found for the plateau model with a dose-varying RBE. It can be concluded that for doses above $1 \mathrm{~Gy}$ there is a tendency for
\end{abstract}

U. Schneider $(\bowtie)$

Division of Medical Physics,

Department of Radiation Oncology and Nuclear Medicine,

The Triemli Hospital Zürich,

8063 Zürich, Switzerland

e-mail: uwe.schneider@psi.ch

L. Walsh

GSF National Research Center,

Institute of Radiation Protection,

85764 Neuherberg, Germany a nonlinear dose-response curve. In addition, there is evidence of a neutron RBE greater than 10 for the A-bomb survivor data. Many problems and uncertainties are involved in combing these two datasets. However, since very little is currently known about the shape of doseresponse relationships for radiation-induced cancer in the radiotherapy dose range, this approach could be regarded as a first attempt to acquire more information on this area. The work presented here also provides the first direct evidence that the bending over of the solid cancer excess risk dose response curve for the A-bomb survivors, generally observed above $2 \mathrm{~Gy}$, is due to cell killing effects.

\section{Introduction}

The dose-response relationship for radiation carcinogenesis up to 1 or 2 Gy has been quantified in several major analyses of the atomic bomb survivors data; recently papers have been published, for example, by Preston et al. [1,2] and Walsh et al. [3, 4]. This dose range is important for radiation protection purposes where low doses are of particular interest. However, it is also important to know the shape of the dose-response curve for radiation-induced cancer for doses above $1 \mathrm{~Gy}$. In patients who receive radiotherapy, parts of the patient volume can receive high doses and it is therefore of great importance to know the risk for the patient to develop a cancer which could have been caused by the radiation treatment. In addition, the health risk to astronauts from space radiation is recognized as one of the limiting factors for long-term space missions. During solar events astronauts can receive doses which are above 1 Gy [5, 6].

The shape of the dose-response curve for radiationinduced cancer is currently of much debate [7-16]. It is not 
known whether cancer risk as a function of dose continues to be linear or decreases at high dose due to cell killing or levels off due to, for example, a balance between cell killing and repopulation effects. The work presented here, aims to clarify the dose-response shape for doses above $1 \mathrm{~Gy}$. In this dose range, data are available from the atomic bomb survivors from Hiroshima and Nagasaki, although the data pertaining to doses above 4 Gy have not usually been included in previous analyses, due to the associated large uncertainties. In addition, data are available from about 30,000 patients with Hodgkin's disease who were irradiated with localized doses of up to around $40 \mathrm{~Gy}$. The aim of this paper is to attempt a combination of the epidemiological data from the atomic bomb survivors and the Hodgkin data, in order to determine a possible doseresponse relationship for radiation-induced solid cancer for radiotherapy doses. Many problems and uncertainties (see "Discussion" section) are involved in combing these two datasets. However, since very little is currently known about the shape of dose-response relationships for radiation-induced cancer in the radiotherapy dose range, this approach could be regarded as a first attempt to acquire more information on this area.

\section{Materials and methods}

Modeling of the atomic bomb survivors data

The most recent data for all solid cancers incidences relating to the follow-up period from 1958 to 1998 and with DS02 doses (which is publicly available from the Radiation Effects Research Foundation-RERF, web site: http://www.rerf.or.jp) were analyzed here [1]. Computations were performed with respect to the organ-averaged weighted doses because reference to the colon weighted dose, which is supplied in the standard dataset for use in the analysis of all solid cancers combined, is known to underestimate the average dose to all organs [17]. The organ-averaged doses are calculated with weighting factors accounting for the risk contribution of individual tumor sites. The weighted organ-averaged doses are greater than the colon doses (which are used in the RERF analyses) by factors of 1.085 and 2 for the gamma and neutron contributions, respectively.

Data of 281 survivors (out of a total of 86,611) with doses varying from 4 to $13 \mathrm{~Gy}$ were included with the main data of doses between 0 and $4 \mathrm{~Gy}$ in the present analysis. The high-dose data were regrouped into organ-averaged absorbed dose categories of 4-6 Gy and 6-13 Gy. All doses were adjusted to allow for random errors in dose estimates by the method of Pierce et al. 1990 [18].
The excess absolute risk (EAR) models were optimized against the epidemiological data, by the maximum likelihood Poisson regression method employing the EPICUREAMFIT software [3]. Although the baseline rates can be dealt with stratification, the main calculations here adopt the fully parametric baseline model of Preston et al. [1].

Since there are some recently discovered indications of a high neutron dose contribution [19, 20], the data were fitted separately for three different values for the relative biological effectiveness of neutrons $(10,35$ and 100). In addition, a dose dependent $\mathrm{RBE}_{\mathrm{D}}$ for neutrons determined by Sasaki et al. [21] using the kerma-weighted chromosomal effectiveness of A-bomb spectrum energy photons was used (Eq. 9 in [21]). When applying Sasaki's equation the maximum RBE of the neutrons at the low-dose limit is 75.1 .

The EAR models applied here were the same as those already considered and explained in detail [1, 2]. Here organ-averaged weighted dose $D$ is the sum of the $\gamma$-ray dose and the RBE-weighted neutron dose. The differences between the previous [2] and present work are that the input data include two extra high-dose categories; organ-average weighted doses are applied instead of colon-weighted doses; a range of neutron weighting factors (RBEs) are considered; and different forms of dose-response relationships, which are more suitable for the high-dose data, i.e. a linear-exponential and a plateau model are employed.

The excess absolute risk is factorized into a function of dose $f(D)$ and a modifying function that depends on the variables gender $(s)$ and age at exposure $(e)$ and age attained $(a)$ :

$\operatorname{EAR}(D, e, a, s)=\beta f(D) \mu(e, a, s)$

where $\beta$ is the initial slope and $\mu$ the modifying function containing the population dependent variables:

$$
\begin{aligned}
& \mu(e, a, s)=\exp \left(\gamma_{e}(e-37)+\gamma_{a} \ln \left(\frac{a}{46}\right)\right)(1 \pm s) \\
& (+ \text { for females },- \text { for males })
\end{aligned}
$$

In this form the fit parameters are gender-averaged and centered at an age at exposure $(e)$ of 37 years and an attained age (a) of 46 years, since these are the characteristic ages of the Hodgkin's patient population as described in the next section. However, the centering is not critical to the fitting procedure and the resulting risks can be scaled to genderspecific values for any values of the two age variables.

It should be noted here that the dose-dependent part $f(D)$ of Eq. (1), which is, to a first approximation, populationindependent is sometimes called organ equivalent dose (OED) [13], when averaged over the whole body volume. For highly inhomogeneous dose distributions, cancer risk is proportional to average dose only for a linear doseresponse relationship. For any other dose-response 
relationship the OED in the body is proportional to cancer risk, if it is defined as

$\mathrm{OED}=\frac{1}{V} \sum_{i} V_{i} f\left(D_{i}\right)$

where $V$ is the total body volume and the sum is taken over all volume elements $V_{i}$ with homogenous dose. The OED is hence a dose-response weighted dose variable, which is proportional to cancer risk in one population (same gender, age at exposure and age attained). This quantity allows comparisons of, e.g. dose distributions in a radiotherapy patient with respect to radiation induced cancer.

Three different dose-response relationships are considered here. The first is a linear response over the whole dose range:

$\mathrm{OED}=\frac{1}{V} \sum_{i} V_{i} D_{i}$

The second is a linear-exponential dose-response relationship of the form:

$\mathrm{OED}=\frac{1}{V} \sum_{i} V_{i} D_{i} \exp \left(-\alpha D_{i}\right)$

and the third is a dose-response, which is flattening at high dose, a so-called plateau dose-response [9] described by:

$\mathrm{OED}=\frac{1}{V} \sum_{i} V_{i} \frac{\left(1-\exp \left(-\delta D_{i}\right)\right)}{\delta}$

All of the dose-response curves defined by Eqs. (4)-(6) become, in the limit of small dose:

$\lim _{D \rightarrow 0} \mathrm{OED}=\frac{1}{V} \sum_{i} V_{i} D_{i}=\bar{D}$

Hence the OED is, in the case of a homogenous distribution of small dose, average absorbed organ dose, which is consistent with radiation protection schemes.

It should be noted here that it is possible to define a homogenous organ dose, OHD, which would result in the same radiation-induced cancer rate as the inhomogenous dose distribution. OHD is then, for the linear-exponential model, simply the inverse function of Eq. (5):

$\mathrm{OHD}=-\frac{1}{\alpha}$ LambertW $(-\alpha \mathrm{OED})$

where Lambert $W$ is the Lambert-function. For a plateau dose-response relationship, the corresponding homogenous dose is:

$\mathrm{OHD}=-\frac{1}{\delta} \ln (1-\delta \mathrm{OED})$

When applying a dose-response model which is linear in dose, even for large doses Eq. (4), the OHD is simply the mean dose.
The data were also test-fitted using a linear-quadraticexponential EAR model. However, the fit parameters relating to the quadratic term in dose were not found to be statistically significantly determined (in contrast to previous results for the lower dose range of $0-2 \mathrm{~Sv}$ for excess relative risk models, [3]) indicating that a linear-exponential dose-response curve may be a better representation of the dose response than a linear-quadratic-exponential dose-response, when the data pertaining to high doses are included in the analysis.

Modeling of the Hodgkin's patients

Cancer risk is only proportional to average organ dose as long as the dose-response curve is linear. At high dose it could be that the dose-response relationship is nonlinear and as a consequence, OED replaces average dose to quantify radiation-induced cancer. In order to calculate OED in radiotherapy patients, information on the three-dimensional dose distribution is necessary. This information is usually not provided in epidemiological studies on second cancers after radiotherapy. However, in Hodgkin's patients the threedimensional dose distribution can be reconstructed.

For this purpose data on secondary cancer incidence rates in various organs for Hodgkin's patients treated with radiation were also included in this analysis. Data on Hodgkin's patients treated with radiation seem to be ideal for an attempted combination with the A-bomb data. These patients were treated at a relatively young age, with curative intent and hence secondary cancer incidence rates for various organs are known with a good degree of precision. Since the treatment of Hodgkin's disease with radiotherapy has been highly successful in the past, the treatment techniques have not been modified very much over the last 30 years. This can be verified, for example, by a comparison of the treatment planning techniques used from 1960 to 1970 [22] with those used from 1980 until 1990 [23]. Additionally, the therapy protocols do not differ very much between the institutions that apply this form of treatment. These factors make it possible to reconstruct a statistically averaged OED distribution for each dose-response model $f(D)$, which is characteristic for a large patient collective of Hodgkin's disease patients.

The overall risk of selected second malignancies of 32,591 Hodgkin's patients after radiotherapy has been quantified by Dores et al. [24]. They found, for all solid cancers after the application of radiotherapy as the only treatment, an excess absolute risk of 39 per 10,000 patients per year (with a 95\% confidence interval ranging from 35.4 to 40.5). The total number of person years in these studies was 92,039 with a mean patient age at diagnosis of 37 years. In combining the Hodgkin data with the A-bomb 
Table 1 Population-dependent variables with one standard deviation in brackets applying the EPICURE-AMFIT code to the atomic survivor data

\begin{tabular}{|c|c|c|c|c|}
\hline & \multicolumn{4}{|l|}{ RBE } \\
\hline & 10 & 35 & 100 & $\mathrm{RBE}_{\mathrm{D}}$ \\
\hline Gender $s$ & $0.1686(0.070)$ & $0.1707(0.070)$ & $0.1742(0.071)$ & $0.1704(0.071)$ \\
\hline Age at exposure $\gamma_{e}$ & $-0.0285(0.006)$ & $-0.0280(0.006)$ & $-0.0273(0.006)$ & $-0.0277(0.006)$ \\
\hline Attained $\gamma_{a}$ & $2.408(0.273)$ & $2.423(0.274)$ & $2.432(0.277)$ & $2.409(0.274)$ \\
\hline
\end{tabular}

survivor data "age at diagnosis of Hodgkin's disease" was equated to "age at exposure to the A-bomb", and "age at diagnosis of Hodgkin's disease + follow-up-time" was equated to "attained age of A-bomb survivors". The follow-up time distribution of the Hodgkin's patients [24] can be used with the temporal patterns of the atomic bomb data of Eq. (2) to obtain the mean attained age ( $a=46$ years). The mean age at exposure and mean attained age for the Hodgkin population was then used to center the fit of the A-bomb survivor data.

In several studies, no increased risk of solid cancers overall was observed after the application of chemotherapy alone. Dores et al. calculated the solid cancer risk both, after radiotherapy alone and combined modality therapy, and found an excess absolute risk of 43 per 10,000 patients per year for the latter. As a consequence, the difference in risk between combined modality treatment and radiotherapy

Table 2 Results of the fit to the atomic bomb data only. In brackets one standard deviation is given

\begin{tabular}{lllll}
\hline RBE & $\begin{array}{l}\text { Model } \\
\text { parameter }\end{array}$ & Linear & $\begin{array}{l}\text { Linear- } \\
\text { exponential }\end{array}$ & Plateau \\
\hline 10 & $\beta^{\mathrm{b}}$ & $9.572(3.84)$ & $14.201(0.95)$ & $14.348(0.94)$ \\
& $\alpha^{\mathrm{a}}$ & - & $0.089(0.024)$ & - \\
& $\delta^{\mathrm{a}}$ & - & - & $0.206(0.027)$ \\
& $P$ value & $>0.5$ & $>0.5$ & $<0.01$ \\
35 & $\beta^{\mathrm{b}}$ & $7.093(4.08)$ & $11.815(0.84)$ & $12.259(0.81)$ \\
& $\alpha^{\mathrm{a}}$ & - & $0.082(0.021)$ & - \\
& $\delta^{\mathrm{a}}$ & - & - & $0.202(0.023)$ \\
& $P$ value & $>0.5$ & $>0.5$ & $<0.01$ \\
100 & $\beta^{\mathrm{b}}$ & $4.030(3.83)$ & $8.365(0.66)$ & $8.873(0.65)$ \\
& $\alpha^{\mathrm{a}}$ & - & $0.066(0.017)$ & - \\
& $\delta^{\mathrm{a}}$ & - & - & $0.178(0.018)$ \\
& $P$ value & $>0.5$ & $>0.5$ & 0.751 \\
$\mathrm{RBE}_{\mathrm{D}}$ & $\beta^{\mathrm{b}}$ & $8.813(2.33)$ & $11.710(0.74)$ & $11.790(0.76)$ \\
& $\alpha^{\mathrm{a}}$ & - & $0.064(0.020)$ & - \\
& $\delta^{\mathrm{a}}$ & - & - & $0.143(0.021)$ \\
& $P$ value & $>0.5$ & 0.477 & $<0.01$
\end{tabular}

The $P$ value was calculated using a $\chi^{2}$-statistic with 21 degrees of freedom

a in $\mathrm{Gy}^{-1}$

b in $(10,000 \text { PY Gy })^{-1}$ alone (4 per 10,000 patients per year) can be tentatively attributed to either chemotherapy or a genetic susceptibility of the Hodgkin patient population with regard to cancer or both. For this reason, we used this risk difference as an error estimate for the subsequent analysis.

A statistically averaged dose distribution was reconstructed, which is characteristic for a large patient collective of Hodgkin's disease patients in the Zubal Phantom, a voxel-based anthropomorphic phantom [25]. Different treatment plans for the various patterns of lymph nodes involvement [26] were obtained. The dose distributions were converted into OED according to Eqs. (4)-(6), assuming a mechanistic approach of cancer risk, where it is assumed that the total risk is the volume-weighted sum of the risks of the partial volumes. A statistically averaged OED distribution was then obtained by combining the OED from different plans with respect to the statistical weight of the involvement of the individual lymph nodes [26]. Details of the treatment plans were taken from the review by Hoppe [23]. The Eclipse External Beam Planning system version 6.5 (Varian Oncology Systems, Palo Alto, CA) was used for treatment planning with corrected dose distributions for head-, phantom- and collimator-scatter also including the extremities. Three different treatment plans were computed which included a mantle field, an inverted$Y$ field and a para-aortic field. All plans were calculated with $6 \mathrm{MV}$ photons and consisted of two opposed fields. The prescribed dose was 36 Gy. The OED representing the risk for all solid cancers was finally determined as the average OED in the whole Zubal phantom.

Combined fit of A-bomb survivor and Hodgkin's patients

Since the dose distribution in a Hodgkin's patient is highly inhomogenous and the dose-response relationships as described by Eqs. (5)and (6) are nonlinear, it is not appropriate to apply a straight forward fit to the data. An iterative fitting procedure needs to be used instead. For this purpose, as described in the last section, the whole threedimensional dose distribution used for Hodgkin treatment was converted into an OED-distribution for given model parameters $\alpha$ or $\delta$. In addition, the dose data of the atomic 
Fig. 1 Plot of cancer incidence per 10,000 persons per year as a function of organ equivalent dose (OED) of the A-bomb survivors (as squares) and the Hodgkin's patients (as a diamond). a, $\mathbf{c}, \mathbf{e}$ and $\mathbf{g}$ show the fit to the A-bomb survivor data using a linear-exponential model and $\mathbf{b}, \mathbf{d}, \mathbf{f}$ and $\mathbf{h}$ using a plateau-dose model. The data and fits are presented for four different neutron RBE models and for age at exposure of 37 years and attained age of 46 years
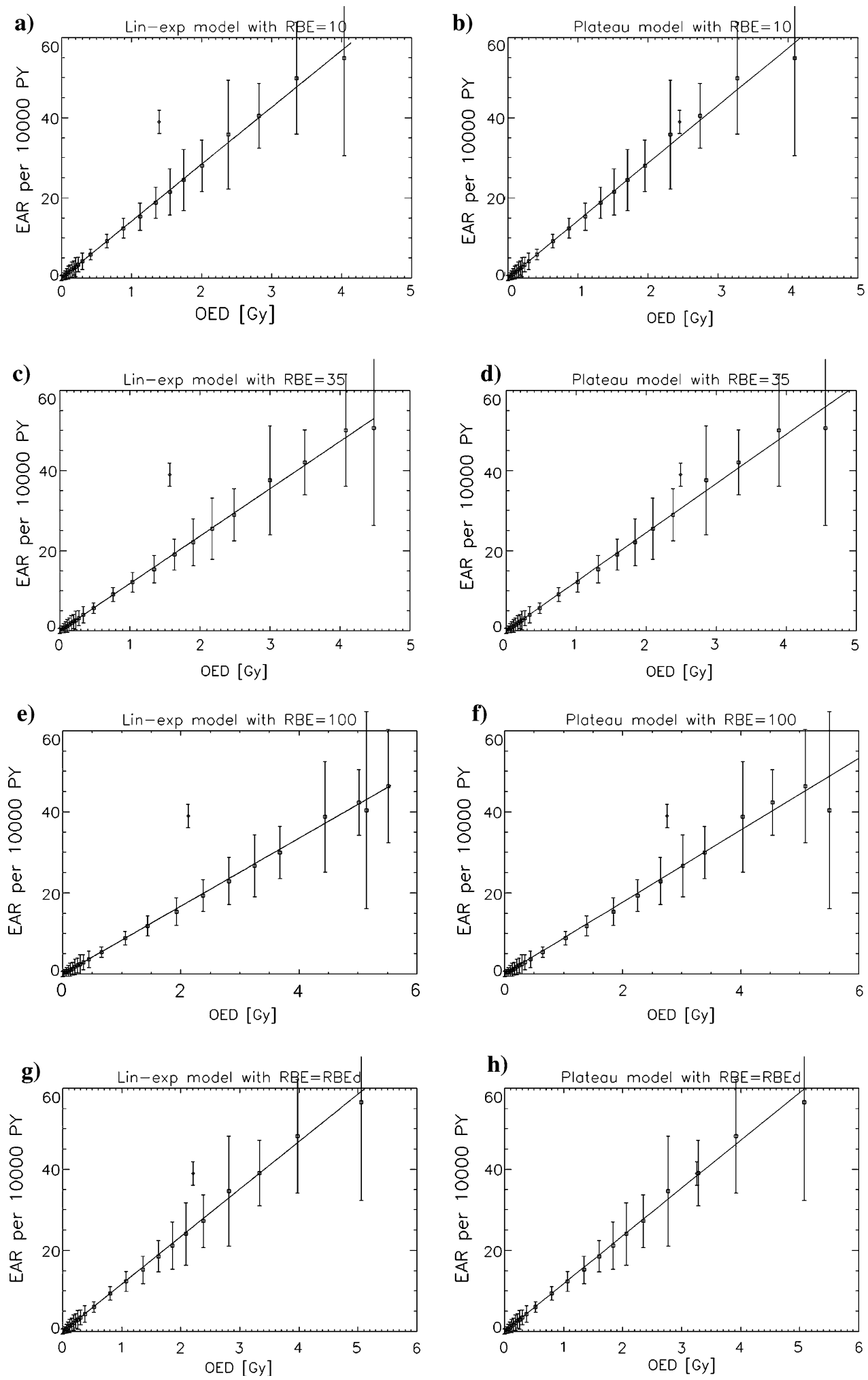

bomb survivors were converted to OED using Eqs. (5) and (6), and a homogenous whole body irradiation of the subjects was assumed. Since EAR as a function of OED is by definition linear Eq. (1), a linear curve was fitted to the combined dataset. The fitted EAR values were compared to the original data and weighted with the inverse of their variances. The $\alpha$ - and $\delta$-values were fitted iteratively by minimizing $\chi^{2}$ 
Table 3 Results of the fit to the Hodgkin's data only

\begin{tabular}{lllll}
\hline RBE & $\begin{array}{l}\text { Model } \\
\text { parameter }\end{array}$ & Linear & $\begin{array}{l}\text { Linear- } \\
\text { exponential }\end{array}$ & Plateau \\
\hline 10 & $\beta^{\mathrm{b}}$ & $3.016(0.31)$ & $14.201(0.82)$ & $14.378(0.90)$ \\
& $\alpha^{\mathrm{a}}$ & - & $0.055(0.018)$ & - \\
& $\delta^{\mathrm{a}}$ & - & - & $0.180(0.023)$ \\
& $P$ value & $>0.5$ & $<0.01$ & $<0.01$ \\
35 & $\beta^{\mathrm{b}}$ & $3.016(0.31)$ & $11.815(0.69)$ & $12.259(0.75)$ \\
& $\alpha^{\mathrm{a}}$ & - & $0.047(0.014)$ & - \\
& $\delta^{\mathrm{a}}$ & - & - & $0.150(0.018)$ \\
& $P$ value & $>0.5$ & $<0.01$ & $<0.01$ \\
100 & $\beta^{\mathrm{b}}$ & $3.016(0.31)$ & $8.365(0.51)$ & $8.873(0.54)$ \\
& $\alpha^{\mathrm{a}}$ & - & $0.034(0.010)$ & - \\
& $\delta^{\mathrm{a}}$ & - & - & $0.100(0.011)$ \\
& $P$ value & $>0.5$ & 0.061 & 0.013 \\
$\mathrm{RBE}_{\mathrm{D}}$ & $\beta^{\mathrm{b}}$ & $3.016(0.31)$ & $11.710(0.70)$ & $11.790(0.75)$ \\
& $\alpha^{\mathrm{a}}$ & - & $0.047(0.018)$ & - \\
& $\delta^{\mathrm{a}}$ & - & - & $0.140(0.020)$ \\
& $P$ value & $>0.5$ & $<0.01$ & $<0.01$ \\
\hline
\end{tabular}

For the linear-exponential and the Plateau-model the initial slope of the atomic bomb data fit is used (Table 2). In brackets one standard deviation is given. The $P$ value was calculated using a $\chi^{2}$-statistic with 22 degrees of freedom

a in $\mathrm{Gy}^{-1}$

b in $(10,000 \text { PY Gy })^{-1}$

$\chi^{2}=\sum\left(\frac{\mathrm{EAR}_{j}-\mathrm{EAR}_{j}^{\mathrm{fit}}}{\sigma_{j}}\right)^{2}$

where $\sigma_{j}$ is the standard deviation of each data point and the sum includes both the A-bomb and the Hodgkin data.

The combination of the A-bomb survivor data with the Hodgkin's patients data made it necessary to use a fitting procedure other than the EPICURE-AMFIT software. To be consistent throughout $\beta, \alpha$ and $\delta$ for the A-bomb survivors data were re-fitted using the above-mentioned fitting routine. Since the re-fitted parameters agree within their standard errors with results using the EPICURE-AMFIT software, only the results of the re-fits are given here.

\section{Results}

Fits to A-bomb survivors data alone

Fitting the EAR model dose modifying function Eq. (2) with EPICURE-AMFIT to the atomic survivor data yields the population-dependent variables listed with the standard deviations in Table 1.

If a simple linear fit is optimized against the data, it is possible to determine the initial slope $\beta$ for a neutron
$\mathrm{RBE}=10,35,100$ and $\mathrm{RBE}_{\mathrm{D}}$. The data are listed together with the corresponding standard deviations and $P$ values in Table 2 . The $P$ values were obtained by applying a $\chi^{2}$ statistic with 21 degrees of freedom applied to the complete dataset (A-bomb survivors and Hodgkin data). Values for $\beta$ for a linear-exponential fit and for plateau-fit to the Abomb survivors are also given in Table 2. The linearexponential and plateau-fits for all four neutron RBE values considered here are plotted in Fig. 1.

Fits to the Hodgkin's data alone

Since in the limit of small dose both, the linear-exponential and the plateau-dose-response curve, as described by Eqs. (5) and (6), respectively, become linear with dose, the initial slope $\beta$ is by definition the same as that required for application with small doses in radiation protection. For this reason, the initial slope is taken from the fits to the Abomb survivors of Table 2. The remaining model parameters $\alpha$ and $\delta$, were then determined by an iterative fit to the Hodgkin's data point. For the linear fit, an initial slope of 3.016 is obtained independently of the neutron RBE. The linear-exponential fit results in $\alpha$ and $\delta$ values, which are listed in Table 3 and plotted in Fig. 2 for the different values of the neutron RBE considered here.

Fits to A-bomb and Hodgkin's data combined

The combined dataset of A-bomb survivors data and Hodgkin's patients were fitted iteratively. The model parameters together with their standard deviations and the $P$ values are listed in Table 4 . The fitted functions are plotted in Fig. 3.

\section{Discussion}

The epidemiological data from the A-bomb survivors and the Hodgkin's patients are associated with large errors as discussed below. Nevertheless, some basic conclusion can be tentatively drawn from the analysis presented here. The quality of the applied fits measured by the $P$ value (listed in Tables 2-4) shows that the linear model does not describe the data as well as the two other models. It seems that for doses above $4 \mathrm{~Gy}$, the dose-response relationship is flattening. However, there is not much difference between the linear-exponential and the plateau-dose-response relationships, regarding their quality of fit. Both models fit the data well with a slight advantage for the plateau model.

It has been observed $[17,27]$ that cancer risks for patients exposed to ionizing radiation in the treatment of 
Fig. 2 Plot of cancer incidence per 10,000 persons per year as a function of organ equivalent dose (OED) of the atomic bomb survivors (as squares) and the Hodgkin's patients (as a diamond). a, c, e and $\mathbf{g}$ show the fit to the Hodgkin's patients using a linear-exponential model and $\mathbf{b}, \mathbf{d}, \mathbf{f}$ and $\mathbf{h}$ using a plateau-dose model. The data and fits are presented for four different neutron RBE models and for age at exposure of 37 years and attained age of 46 years
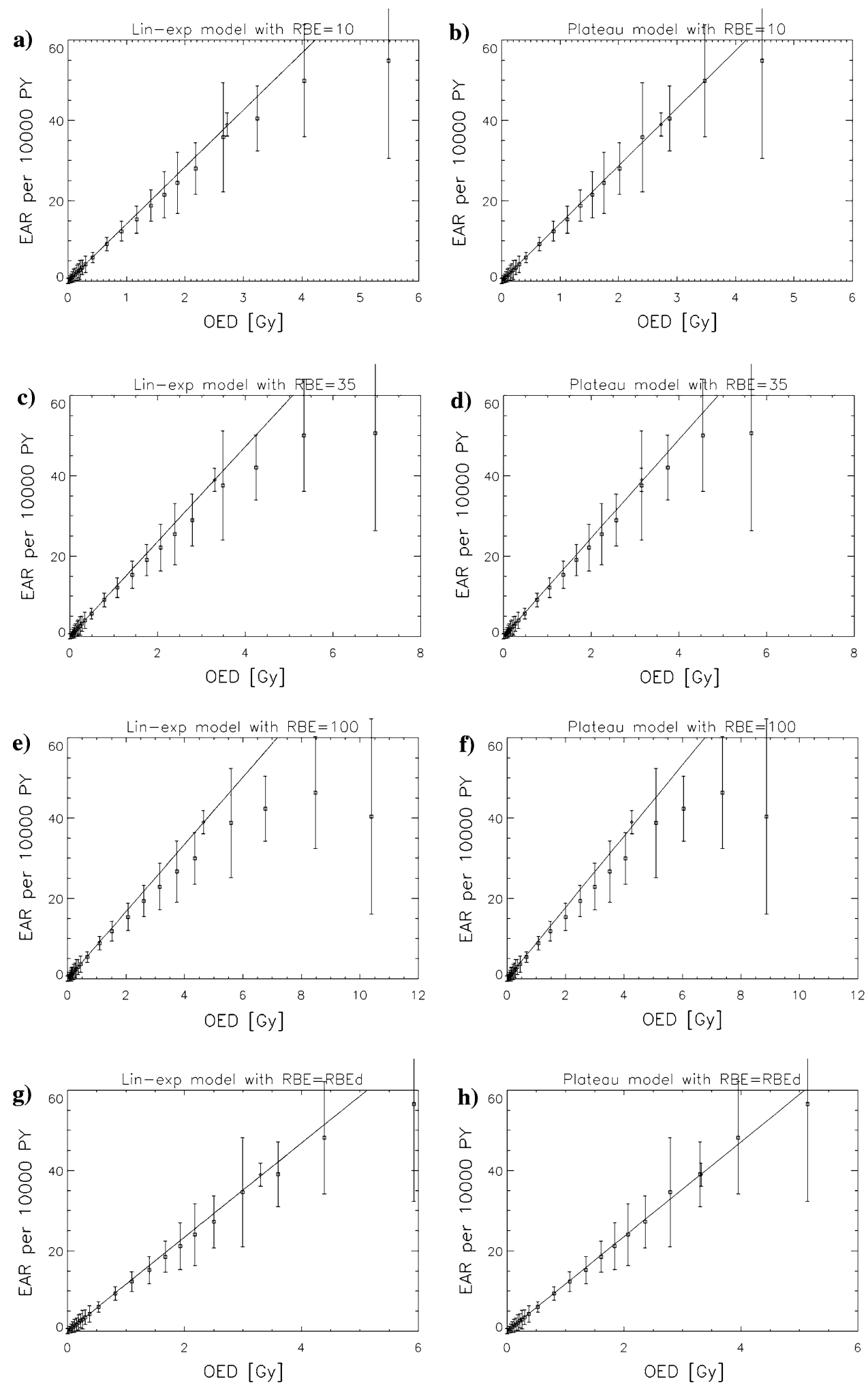

cancer, are generally lower (when plotted against average absorbed dose, not OED) than those estimated from the A-bomb survivors. It was suggested that cell sterilization, dose fractionation or a larger neutron RBE in the A-bomb data could account for this difference. The present analysis using OED, which includes cell sterilization effects, shows good agreement with the A-bomb data which are plotted as a function of absorbed dose for a RBE of 10 
Table 4 Results of the fit to the atomic bomb data and the Hodgkin's data combined

\begin{tabular}{lllll}
\hline RBE & $\begin{array}{l}\text { Model } \\
\text { parameter }\end{array}$ & Linear & $\begin{array}{l}\text { Linear- } \\
\text { exponential }\end{array}$ & Plateau \\
\hline 10 & $\beta^{\mathrm{b}}$ & $4.170(9.01)$ & $12.367(0.81)$ & $13.824(0.90)$ \\
& $\alpha^{\mathrm{a}}$ & - & $0.050(0.018)$ & - \\
& $\delta^{\mathrm{a}}$ & - & - & $0.179(0.023)$ \\
& $P$ value & $>0.5$ & $<0.01$ & $<0.01$ \\
35 & $\beta^{\mathrm{b}}$ & $4.140(6.86)$ & $9.877(0.68)$ & $10.966(0.75)$ \\
& $\alpha^{\mathrm{a}}$ & - & $0.043(0.014)$ & - \\
& $\delta^{\mathrm{a}}$ & - & - & $0.146(0.018)$ \\
& $P$ value & $>0.5$ & $<0.01$ & $<0.01$ \\
100 & $\beta^{\mathrm{b}}$ & $3.522(4.20)$ & $6.689(0.51)$ & $7.205(0.56)$ \\
& $\alpha^{\mathrm{a}}$ & - & $0.034(0.010)$ & - \\
& $\delta^{\mathrm{a}}$ & - & - & $0.111(0.012)$ \\
& $P$ value & $>0.5$ & 0.053 & 0.168 \\
$\mathrm{RBE}_{\mathrm{D}}$ & $\beta^{\mathrm{b}}$ & $4.184(6.72)$ & $10.774(0.70)$ & $11.677(0.70)$ \\
& $\alpha^{\mathrm{a}}$ & - & $0.044(0.017)$ & - \\
& $\delta^{\mathrm{a}}$ & - & - & $0.139(0.019)$ \\
& $P$ value & $>0.5$ & $<0.01$ & $<0.01$
\end{tabular}

In brackets one standard deviation is given. The $P$ value was calculated using a $\chi^{2}$-statistic with 22 degrees of freedom

a in $\mathrm{Gy}^{-1}$

b in $(10,000 \text { PY Gy })^{-1}$

and for Sasaki's RBE in Fig. 4. It has often been hypothesized that the bending-over, of the solid cancer excess risk dose-response curve for the A-bomb survivors, that has been observed to occur above $2 \mathrm{~Gy}$, could be due to cell killing effects. The work presented here might provide the first direct evidence for this. The impact of dose fractionation and repopulation is not included in the present analysis.

The average doses in the two highest-weighted dose categories are increased from 5.4 and $8.9 \mathrm{~Sv}$ for a RBE 10 up to 12.7 and $22.1 \mathrm{~Sv}$ for a RBE of 100 , respectively. Since the data in these high dose categories are subject to very large errors (standard deviation 0.8 and $2.3 \mathrm{~Sv}$ for $\mathrm{RBE}=10$, and 6.1 and $12.3 \mathrm{~Sv}$ for $\mathrm{RBE}=100$ ), it is not possible to assess the degree of dependability of the assumption of a large neutron RBE values such as 100, if the mean doses in these dose categories are compared with the lethal doses for humans (LD50). Additionally, it is worth noting that the last dose category employed here has been omitted in all previous analyses of these data, since the small chance of survival suggests that estimates of doses in this upper group could possibly be too large [18]. Sasaki's formulation of a neutron RBE which is variable with dose results in a dose-response curve which fits the data well and the average dose in the two highest dose categories is only increased to 5.7 and $9.0 \mathrm{~Sv}$. This could be an additional indication for a dose-dependent neutron RBE.

Increased risks of solid cancers after Hodgkin's disease have been generally attributed to radiotherapy. An important question is whether chemotherapy for Hodgkin's disease also adds to the solid cancer risk, and if so, at which sites. If chemotherapy indeed affects induction of solid tumors, one would expect that patients receiving combined modality treatment would have a greater relative risk than patients treated with radiotherapy alone. Only one study has reported a significantly greater risk for solid cancers overall after combined chemo- and radiotherapy compared with irradiation alone [28], whereas no such difference has been found in the majority of investigations [28]. However, for selected solid cancer sites larger (e.g. lung) or lower (e.g. breast) risks were observed after combined modality treatment than after irradiation alone [28]. It can be tentatively hypothesized, for the analysis presented here, that cancer risk after chemotherapy of disparate sites is balanced in such a way that the risk for all solid tumors is not affected [28].

It is well known that genetic susceptibility underlies Hodgkin's disease [29]. It is not clear whether this genetic susceptibility would also affect the development of other cancers. There is the possibility of a cancer diathesis, the prospect that, for some reasons related to genetic makeup, a person who developed one cancer has an inherently increased risk of developing another. However, such cancer susceptibility would result in a minimal excess cancer incidence compared to the incidence of radiation related tumors, since such an excess cancer incidence of solid tumors should also be seen in Hodgkin's patients after treatment with chemotherapy alone. However, there is no statistically significant increase for all solid tumors combined. Therefore, such an effect will be hidden in the $95 \%$ confidence interval of the observed cancer incidence after chemotherapy.

In this work, EAR has been used to quantify radiationinduced cancer. Usually ERR is recommended for transferring risk from the Japanese population to other populations. EAR is used here, since the risk calculations of the Hodgkin's cohort are based on extremely inhomogenous dose distributions. It is assumed that the total absolute risk in the whole body is the volume-weighted sum of the risks of the partial volumes which are irradiated homogenously. Currently, there is no available method for obtaining analogous whole-body risk using ERR. Since the difference between the Japanese and the US population in EAR for all solid tumors is less than $10 \%$ and only all solid tumors together were analyzed here, the use of EAR is probably justifiable. 
Fig. 3 Plot of cancer incidence per 10,000 persons per year as a function of organ equivalent dose (OED) of the atomic bomb survivors (as squares) and the Hodgkin's patients (as a diamond). a, $\mathbf{c}, \mathbf{e}$ and $\mathbf{g}$ show the fit to the combined dataset of $A$ bomb survivor data and Hodgkin's patients using a linear-exponential model and $\mathbf{b}$, $\mathbf{d}, \mathbf{f}$ and $\mathbf{h}$ using a plateau-dose model. The data and fits are presented for four different neutron RBE models and for age at exposure of 37 years and attained age of 46 years
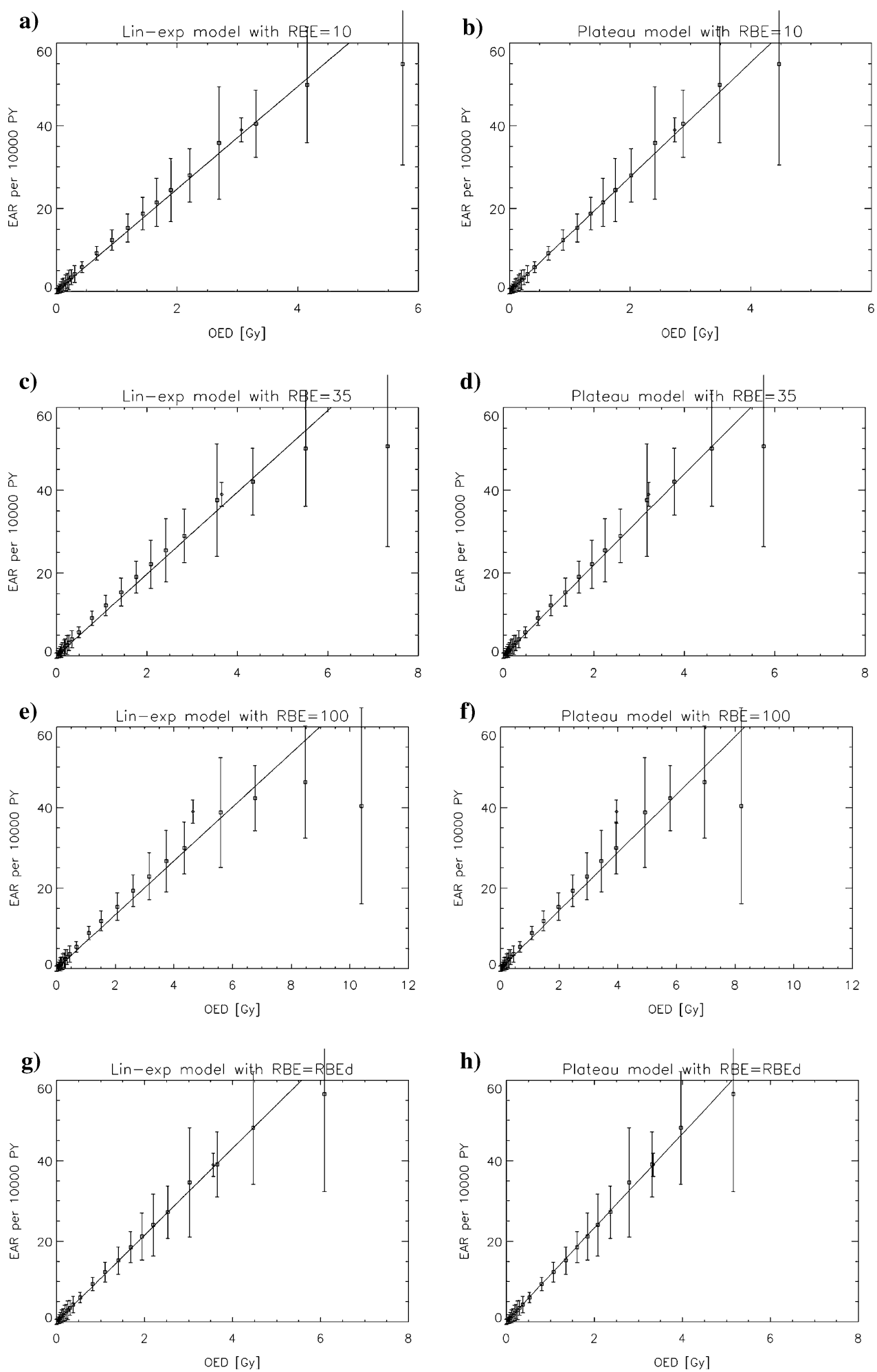

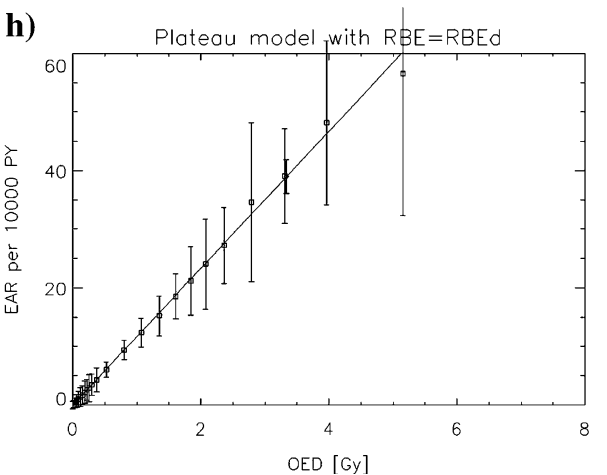

\section{Conclusions}

A comparison of dose distributions in humans, for example in radiotherapy treatment planning, with regard to cancer incidence or mortality can be performed by computing
OED, which can be based on any dose-response relationship. In this work, OED was defined for a linear Eq. (4), a linear-exponential Eq. (5) and a plateau dose-response relationship Eq. (6). The model parameters ( $\alpha$ and $\delta$ ) were obtained by a fit of these OED models to A-bomb survivors 

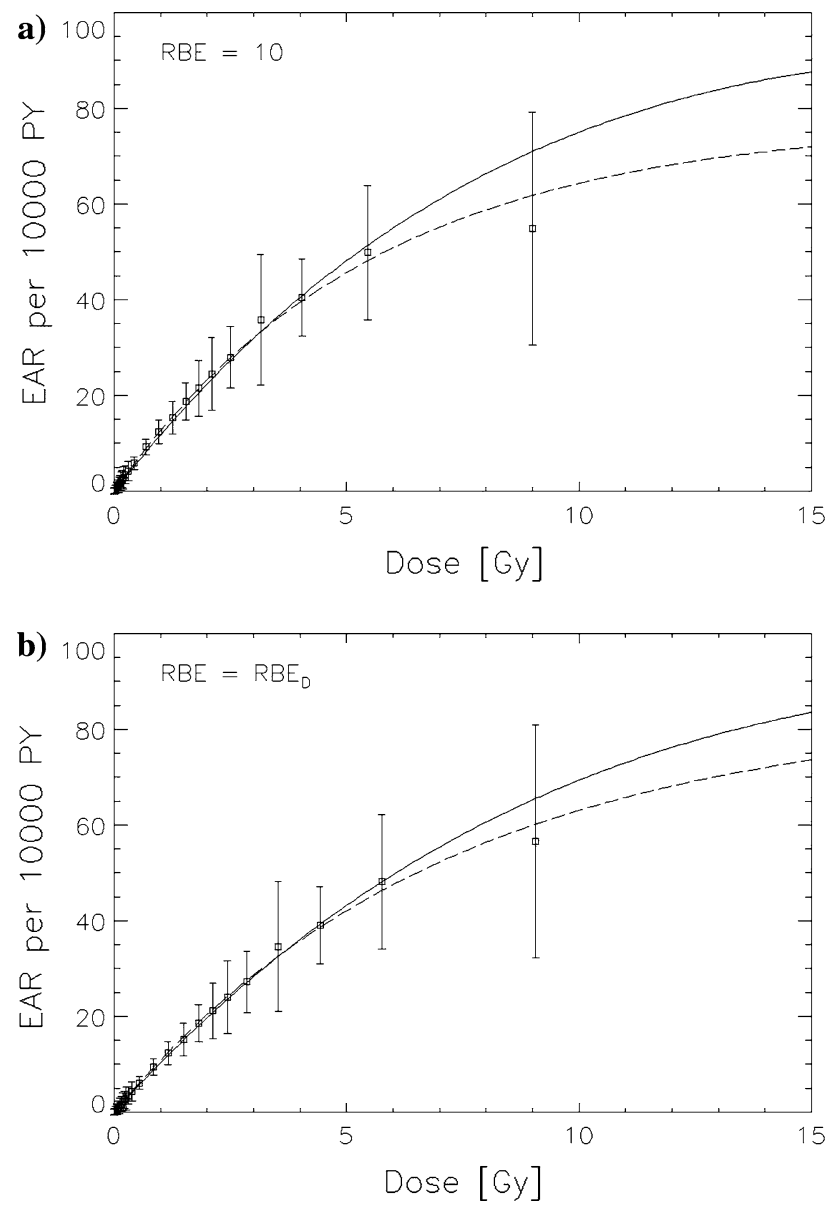

Fig. 4 Plot of cancer incidence per 10,000 persons per year as a function of organ absorbed dose of the atomic bomb survivors (as squares). Shown are the fits to the combined dataset of A-bomb survivor data and Hodgkin's patients using a linear-exponential model (solid line) and a plateau-dose model (dashed line). The data and fits are presented for a neutron RBE of 10 (a) and for a varying $\mathrm{RBE}_{\mathrm{D}}(\mathbf{b})$

and Hodgkin's patients data and are listed in Table 3. For any three-dimensional inhomogenous dose distribution, cancer risk can be compared by computing OED using the coefficients obtained in this work.

For absolute risk estimates, EAR can be determined by taking additionally the fitted initial slope $\beta$ from Table 3 and multiplying it with the population-dependent modifying function Eq. (1) using the fitted coefficients of Table 1. However, absolute risk estimates must be viewed with care, since the errors involved are large.

It has often been hypothesized that the bending over, of the solid cancer excess risk dose response curve for the Abomb survivors, that has been observed to occur above 2 Gy, could be due to cell killing effects. The work presented here might provide the first direct evidence for this.

Acknowledgments Special thanks are due to Dr. Werner Rühm for many useful comments and valuable discussions. This work makes use of the data obtained from the Radiation Effects Research Foundation (RERF) in Hiroshima, Japan. RERF is a private foundation funded equally by the Japanese Ministry of Health and Welfare and the US Department of Energy through the US National Academy of Sciences. The conclusions in this work are those of the authors and do not necessarily reflect the scientific judgment of RERF or its funding agencies.

\section{References}

1. Preston DL, Ron E, Tokuoka S, Funamoto S, Nishi N, Soda M, Mabuchi K, Kodama K (2007) Solid cancer incidence in atomic bomb survivors: 1958-1998. Radiat Res 168:1-64

2. Preston DL, Pierce DA, Shimizu Y, Cullings HM, Fujita S, Funamoto S, Kodama K (2004) Effects of recent changes in Atomic bomb survivor dosimetry on cancer mortality risk estimated. Radiat Res 162:377-389

3. Walsh L, Rühm W, Kellerer AM (2004) Cancer risk estimates for $\mathrm{X}$-rays with regard to organ specific doses, part I: all solid cancers combined. Radiat Environ Biophys 43:145-151

4. Walsh L, Rühm W, Kellerer AM (2004) Cancer risk estimates for $\gamma$-rays with regard to organ specific doses, part II: site specific solid cancers. Radiat Environ Biophys 43:225-231

5. Cucinotta FA, Wu H, Shavers M, George K (2003) Radiation dosimetry and biophysical models of space radiation effects. Gravit Space Biol Bull 16(2):11-18

6. Kim MH, Cucinotta FA, Wilson JW (2007) A temporal forecast of radiation environments for future space exploration missions. Radiat Environ Biophys 46(2):95-100

7. Lindsay KA, Wheldon EG, Deehan C, Wheldon TE (2001) Radiation carcinogenesis modelling for risk of treatment-related second tumours following radiotherapy. $\mathrm{Br} \quad \mathrm{J}$ Radiol 74(882):529-536

8. Hall EJ, Wuu CS (2003) Radiation-induced second cancers: the impact of 3D-CRT and IMRT. Int J Radiat Oncol Biol Phys 56(1):83-88

9. Davis RH (2004) Production and killing of second cancer precursor cells in radiation therapy. Int J Radiat Oncol Biol Phys 59(3):916

10. Schneider U (2005) Dose-response relationship for radiationinduced cancer-decrease or plateau at high dose. Int J Radiat Oncol Biol Phys 61(1):312-313

11. Dasu A, Toma-Dasu I (2005) Dose-effect models for riskrelationship to cell survival parameters. Acta Oncol 44(8):829835

12. Dasu A, Toma-Dasu I, Olofsson J, Karlsson M (2005) The use of risk estimation models for the induction of secondary cancers following radiotherapy. Acta Oncol 44(4):339-347

13. Schneider U, Zwahlen D, Ross D, Kaser-Hotz B (2005) Estimation of radiation induced cancer from 3D-dose distributions: concept of organ equivalent dose. Int J Radiat Oncol Biol Phys 61: $1510-1515$

14. Schneider U, Kaser-Hotz B (2005) A simple dose-response relationship for modelling secondary cancer incidence after radiotherapy. Z Med Phys 15(1):31-37

15. Sachs RK, Brenner DJ (2005) Solid tumor risks after high doses of ionizing radiation. Proc Natl Acad Sci USA 102(37):13040 13045

16. Schneider U, Kaser-Hotz B (2005) Radiation risk estimates after radiotherapy: application of the organ equivalent dose concept to plateau dose-response relationships. Radiat Environ Biophys 44(3):235-2399

17. Kellerer AM, Walsh L (2001), Risk estimation for fast neutrons with regard to solid cancer. Radiat Res 156:708-717 
18. Pierce DA, Stram DO, Vaeth M (1990) Allowing for random errors in radiation dose estimates for the atomic bomb survivor data. Radiat Res 123:275-284

19. Kellerer AM, Rühm W, Walsh L (2006) Indications of the neutron effect contribution in the solid cancer data of the A-bomb survivors. Health Phys 90(6):554-564

20. Rühm W, Walsh L (2007) Current risk estimates based on the Abomb survivors data-a discussion in terms of the ICRP recommendations on the neutron weighting factor. Radiat Prot Dosimetry. (Advance access, May 28, doi:10.1093/rpd/ncm087)

21. Sasaki MS, Endo S, Ejima Y, Saito I, Okamura K, Oka Y, Hoshi M (2006) Effective dose of A-bomb radiation in Hiroshima and Nagasaki as assessed by chromosomal effectiveness of spectrum energy photons and neutrons. Radiat Environ Biophys 45:79-91

22. Carmel RJ, Kaplan HS (1976) Mantle irradiation in Hodgkin's disease. An analysis of technique, tumor eradication, and complications. Cancer 37(6):2813-2825

23. Hoppe RT (1990) Radiation therapy in the management of Hodgkin's disease. Semin Oncol 17(6):704-715

24. Dores GM, Metayer C, Curtis RE, Lynch CF, Clarke EA, Glimelius B, Storm H, Pukkala E, van Leeuwen FE, Holowaty EJ, Andersson M, Wiklund T, Joensuu T, van't Veer MB, Stovall M, Gospodarowicz M, Travis LB (2002) Second malignant neoplasms among long-term survivors of Hodgkin's disease: a population-based evaluation over 25 years. J Clin Oncol 20(16):3484-3494

25. Zubal IG, Harrell CR, Smith EO, Rattner Z, Gindi G, Hoffer PB (1994) Computerized three-dimensional segmented human anatomy. Med Phys 21(2):299-302

26. Mauch PM, Leslie AK, Kadin M, Coleman CN, Osteen R, Hellman S (1993) Patterns of presentation of Hodgkin disease. Cancer 71(6):2062-2071

27. Little MP (2001) Comparison of the risks of cancer incidence and mortality following radiation therapy for benign and malignant disease with the cancer risks observed in the Japanese A-bomb survivors. Int J Radiat Biol 77(4):431-464 (Erratum in: Int J Radiat Biol 2001 77(6):745-60)

28. van Leeuwen FE, Travis LB (2005) Risk of second malignancy in patients with selected primary cancers. In: DeVita VT, Hellman S, Rosenberg SA (eds) Cancer: principles \& practice of oncology, 7th edn. Lippincott Williams \& Wilkins, Philadelphia, pp 25752602

29. Mack TM, Cozen W, Shibata DK et al (1995) Concordance for Hodgkin's disease in identical twins suggesting genetic susceptibility to the young-adult form of the disease. N Engl J Med 332(7):413-418 